\title{
Accurate and Efficient Analysis of Printed Reflectarrays With Arbitrary Elements Using Higher-Order Hierarchical Legendre Basis Functions
}

Zhou, Min; Jørgensen, Erik; Kim, Oleksiy S.; Sorensen, Stig B.; Meincke, Peter; Breinbjerg, Olav

Published in:

I E E E Antennas and Wireless Propagation Letters

Link to article, DOI:

10.1109/LAWP.2012.2208174

Publication date:

2012

Document Version

Publisher's PDF, also known as Version of record

Link back to DTU Orbit

Citation (APA):

Zhou, M., Jørgensen, E., Kim, O. S., Sorensen, S. B., Meincke, P., \& Breinbjerg, O. (2012). Accurate and Efficient Analysis of Printed Reflectarrays With Arbitrary Elements Using Higher-Order Hierarchical Legendre Basis Functions. I E E E Antennas and Wireless Propagation Letters, 11, 814-817.

https://doi.org/10.1109/LAWP.2012.2208174

\section{General rights}

Copyright and moral rights for the publications made accessible in the public portal are retained by the authors and/or other copyright owners and it is a condition of accessing publications that users recognise and abide by the legal requirements associated with these rights.

- Users may download and print one copy of any publication from the public portal for the purpose of private study or research.

- You may not further distribute the material or use it for any profit-making activity or commercial gain

- You may freely distribute the URL identifying the publication in the public portal 


\title{
Accurate and Efficient Analysis of Printed Reflectarrays With Arbitrary Elements Using Higher-Order Hierarchical Legendre Basis Functions
}

\author{
Min Zhou, Student Member, IEEE, Erik Jørgensen, Member, IEEE, Oleksiy S. Kim, Stig B. Sørensen,
} Peter Meincke, Member, IEEE, and Olav Breinbjerg, Member, IEEE

\begin{abstract}
It is demonstrated that nonsingular higher-order hierarchical Legendre basis functions are capable of accounting for the singularities of the electric currents at the edges of the reflectarray elements, thus yielding good convergence properties and very accurate results. In addition, the number of Floquet harmonics needed in the spectral domain method of moments is reduced by using higher-order hierarchical Legendre basis functions as compared to singular basis functions. At the same time, higher-order hierarchical Legendre basis functions can be applied to any arbitrarily shaped array elements, thus providing the flexibility required in the analysis of printed reflectarrays. A comparison to DTU-ESA Facility measurements of a reference offset reflectarray shows that higher-order hierarchical Legendre basis functions produce results of the same accuracy as those obtained using singular basis functions.
\end{abstract}

Index Terms-Accurate antenna analysis, basis functions, Floquet harmonics, method of moments (MoM), reflectarray.

\section{INTRODUCTION}

$\mathbf{P}$ RINTED reflectarrays are becoming viable alternatives to reflector antennas, and they are the subject of increasing research interest [1]. In the analysis and design of reflectarrays, the commonly adopted technique for the calculation of the electric currents on the printed array elements is based on the Local Periodicity approach, where each array element is analyzed assuming that it is located in an infinite array of identical elements [2]. The periodic problem is usually formulated in terms of an integral equation and solved by the spectral domain method of moments (SDMoM) [3]. The Green's function in the integral equation consists of a double summation of Floquet harmonics.

To ensure an accurate yet efficient analysis, suitable basis functions must be selected to minimize the number of basis functions $\left(N_{\mathrm{b}}\right)$ and Floquet harmonics $\left(N_{\mathrm{f}}\right)$. For canonically

Manuscript received June 14, 2012; accepted July 05, 2012. Date of publication July 11, 2012; date of current version July 20, 2012. The reflectarray antenna production and measurement was supported by the European Space Agency under ESTEC Contract No. 4000101041

M. Zhou is with TICRA, Copenhagen 1201, Denmark, and also with the Department of Electrical Engineering, Electromagnetic Systems, Technical University of Denmark, Kgs. Lyngby 2800, Denmark (e-mail: mz@ticra.com).

E. Jørgensen, S. B. Sørensen, and P. Meincke are with TICRA, Copenhagen 1201, Denmark (e-mail: ticra@ticra.com).

O. S. Kim and O. Breinbjerg are with the Department of Electrical Engineering, Electromagnetic Systems, Technical University of Denmark, Kgs. Lyngby 2800, Denmark (e-mail: osk@elektro.dtu.dk; ob@elektro.dtu.dk).

Color versions of one or more of the figures in this letter are available online at http://ieeexplore.ieee.org.

Digital Object Identifier 10.1109/LAWP.2012.2208174 shaped array elements, e.g., rectangular patches, entire domain basis functions with the correct edge conditions, reproducing the singular behavior of the electric currents at the edges, are known for providing fast convergence in the SDMoM with respect to $N_{\mathrm{b}}$ [4], [5]. However, due to their singular behavior, the Fourier spectrum is wide, which increases $N_{\mathrm{f}}$. For arbitrarily shaped elements, the common choices are first-order basis functions, e.g., Rao-Wilton-Glisson (RWG) [6] or rooftop [7] basis functions. However, $N_{\mathrm{b}}$ is high for these cases.

The objective of this letter is to present the use of nonsingular higher-order hierarchical Legendre basis functions as described in [8] in the analysis of printed reflectarrays. The higher-order hierarchical Legendre basis functions can be applied to any arbitrarily shaped elements and, at the same time, maintain a good compromise between $N_{\mathrm{b}}$ and $N_{\mathrm{f}}$.

The Fourier transforms of all the basis functions used in this letter are closed-form expressions.

\section{HighER-ORDER HieRARCHICAL LEGENDRE BASIS FUNCTIONS}

The higher-order hierarchical Legendre basis functions (LegBF) [8] are subdomain nonsingular basis functions. For array elements where the shape can be described using a single mesh element, e.g., rectangular patches, the LegBF can be defined on the entire patch, thus becoming entire domain basis functions.

The LegBF do not possess the singularity behaviors on the edges of the array elements. However, they have - contrary to first-order basis functions, e.g., rooftop basis functions- - good convergence properties. This is illustrated in Fig. 1, where the relative error of the magnitude of the reflection coefficient of a square patch in a periodic environment is displayed. The reflection coefficient for a normally incident plane wave is calculated at $9.6 \mathrm{GHz}$ and displayed as a function of the total $N_{\mathrm{b}}$ on the patch. Five different basis functions are employed: rooftops, subdomain LegBF (S-LegBF), entire-domain LegBF (E-LegBF), the entire-domain singular basis functions weighted by sinusoidal functions (E-SinBF) from [9], and the entire-domain singular basis functions weighted by Chebyshev polynomials of first and second kind (E-CheBF) from [4]. For the S-LegBF, the square patch is divided into $2 \times 2$ mesh elements. The substrate dielectric constant and thickness are $\epsilon_{\mathrm{r}}=3.66$ and $h=0.762 \mathrm{~mm}$, respectively, and the loss $\operatorname{tangent}$ is $\tan \delta=0.0037$. The dimension of the unit cell is $d^{2}=13.5 \times 13.5 \mathrm{~mm}^{2}$, and the square patch is at resonance 


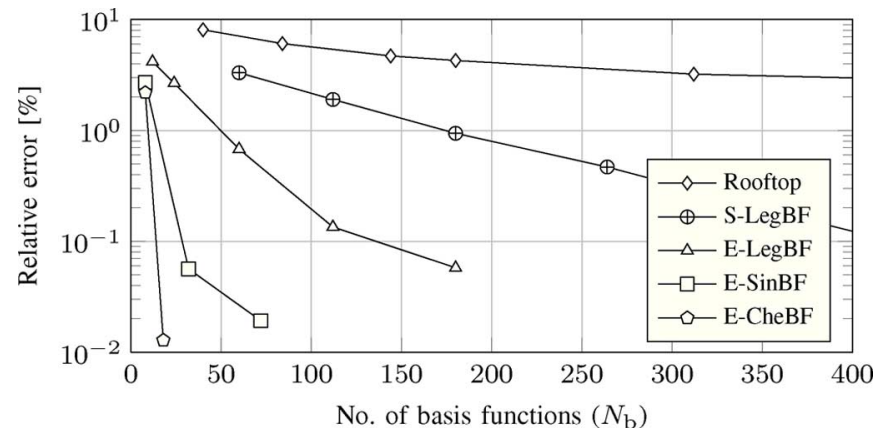

Fig. 1. Relative error of the magnitude of the reflection coefficient of a square patch in a periodic environment as a function of the number of basis functions $N_{\mathrm{b}}$.

with a side length of $7.65 \mathrm{~mm}$. As a reference, $512 \mathrm{E}-\mathrm{CheBF}$ are used.

It is seen that a relative error below $0.1 \%$ is achieved by using $32 \mathrm{E}-\mathrm{SinBF}$ and only $18 \mathrm{E}-\mathrm{CheBF}$. For the same accuracy, $180 \mathrm{E}-\mathrm{LegBF}$ and $480 \mathrm{~S}-\mathrm{LegBF}$ are required. For the rooftops, a relative error below $0.1 \%$ cannot be obtained due to the high $Q$-factor of the patch. The same convergence issues exist for conventional nonsingular entire-domain basis function, e.g., trigonometric basis functions [9]. As expected, $N_{\mathrm{b}}$ required for the LegBF is higher compared to the singular basis functions. However, it demonstrates their superior convergence capabilities compared to the first-order basis functions.

Usually, the double summation of the Floquet harmonics is truncated according to [3]

$$
\sum_{n=-N}^{N} \sum_{m=-M}^{M}
$$

where $n, m$ are the indices for the Floquet harmonics. The main contribution in this summation originates from the combinations of the lower-order Floquet harmonics, thus an appropriate choice for $M$ is $M=N-|n|$, yielding a total number of Floquet harmonics of $N_{\mathrm{f}}=2\left(N^{2}+N\right)+1$. This choice of $M$ corresponds to summing the Floquet harmonics within a rhombus instead of a square when $M=N$. To ensure convergent results in Fig. 1, the number of Floquet harmonics used in the SDMoM calculations has been overestimated. For E-SinBF and E-CheBF, approximately 24000 Floquet harmonics are used, whereas approximately 3300 are used for the rooftops and the LegBF.

Although the convergence rate of the LegBF with respect to $N_{\mathrm{b}}$ is lower compared to the singular basis functions, the convergence rate with respect to $N_{\mathrm{f}}$ is better. Let us define the spatial support of the basis function as $a$ and the spectral variable as $k$, then for a fixed $a$, the Fourier spectrum of the LegBF decays as $1 /(k a)$, whereas it decays as $1 / \sqrt{k a}$ for the singular basis functions. As a result, the Fourier spectrum is narrower for the LegBF, thus decreasing $N_{\mathrm{f}}$.

Using the same test case as for Fig. 1, the relative error of the magnitude of the reflection coefficient as function of $N_{\mathrm{f}}$ is displayed in Fig. 2. A relative error below $0.1 \%$ is desired, thus $18 \mathrm{E}-\mathrm{CheBF}, 32 \mathrm{E}-\mathrm{SinBF}, 180 \mathrm{E}-\mathrm{LegBF}$, and $480 \mathrm{~S}-\mathrm{LegBF}$ are used. It is seen that the required accuracy is obtained using

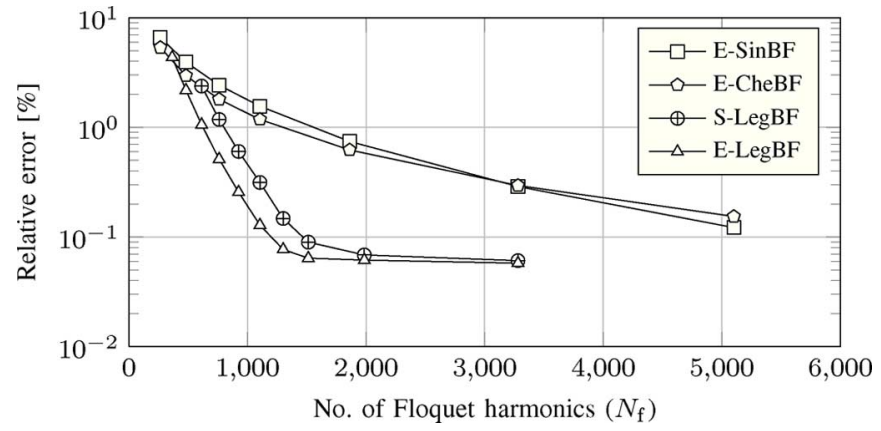

Fig. 2. Relative error of the magnitude of the reflection coefficient of a square patch in a periodic environment as a function of the number of Floquet harmonics $N_{\mathrm{f}}$.

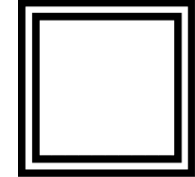

(a)

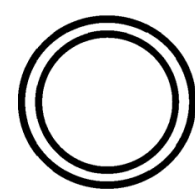

(d)

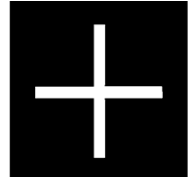

(b)

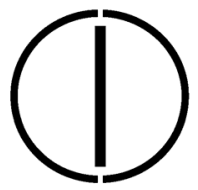

(e)

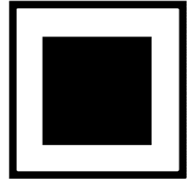

(c)

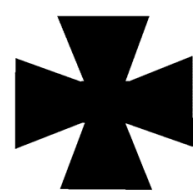

(f)
Fig. 3. (a)-(f) Different element shapes used for reflectarray applications.

approximately 1300 and 2000 Floquet harmonics for E-LegBF and S-LegBF, respectively, whereas over 5000 Floquet harmonics are needed for E-SinBF and E-CheBF.

There exist several acceleration techniques for the efficient computation of (1), e.g., the application of Kummer's transformation [10]-[12], and the two-dimensional fast Fourier transform (2-D FFT) technique [3]. In Kummer's transformation, an asymptotic part is subtracted from the Floquet summation, resulting in a fast converging summation, and the summation of the asymptotic part is treated separately in an efficient way. However, the treatment of the asymptotic part depends on the choice of basis function and the element shape, thus making the technique unsuitable for arbitrarily shaped elements. The 2-D FFT technique is another efficient way of computing the double summation of (1), but it is restricted to basis functions that are defined in a uniform rectangular mesh, thus not applicable for arbitrarily shaped elements. This is a limitation for the analysis of arbitrary reflectarrays, as different element shapes are used for different applications.

The choice of element shape is heavily dictated by requirements such as bandwidth and polarization, and much research has been carried out to investigate the performance of different element shapes [13]-[18]; see Fig. 3. Suitable entire-domain basis functions can be defined only for some of the element shapes, e.g., the concentric circular loops [Fig. 3(d)]. Rooftops and RWG basis functions can be used on arbitrary-shaped elements, but at the cost of high $N_{\mathrm{b}}$. The LegBF are defined on higher-order curvilinear mesh elements [8], hence any curved boundary can be modeled very accurately. Thus, the LegBF can 


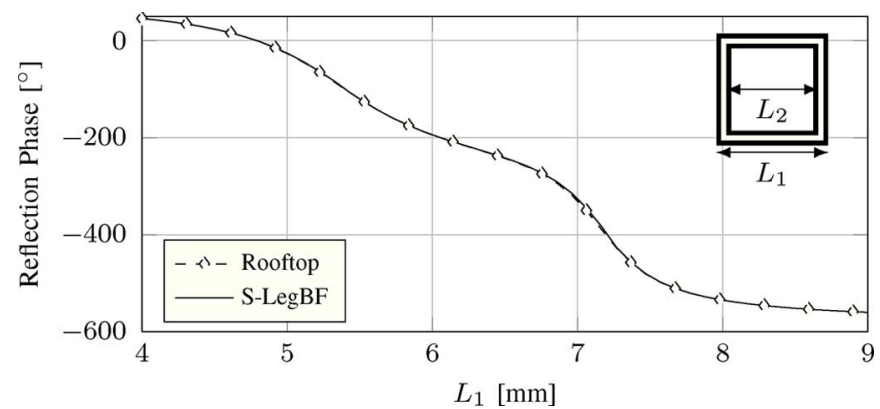

Fig. 4. Phase of the reflection coefficient of concentric square loops in a periodic environment as function of the outer loop length $L_{1}$. The inner loop length is $L_{2}=0.75 L_{1}$; the widths of the loops are $w=0.35 \mathrm{~mm}$.

be applied to any of those element shapes and with a good efficiency. As an example, the phase of the reflection coefficient of the concentric square loop [Fig. 3(a)] in a periodic environment is shown in Fig. 4. The reflection coefficient for a normally incident plane wave is calculated at $9.6 \mathrm{GHz}$ using rooftops and $\mathrm{S}-\mathrm{LegBF}$, and the phase is displayed as function of the outer loop length $L_{1}$. The inner loop length is $L_{2}=0.75 L_{1}$, and the widths of the loops are $w=0.35 \mathrm{~mm}$. The setup is the same as in Figs. 1 and 2, but the substrate thickness is increased to $h=3.175 \mathrm{~mm}$. This is to ensure convergence of the rooftops by reducing the $Q$-factor of the printed element. The phase curve is obtained using a total of 80 rooftops, whereas only $28 \mathrm{~S}-\mathrm{LegBF}$ are required for the same accuracy. For both cases, $N_{\mathrm{f}}=1861$. A similar reduction in $N_{\mathrm{b}}$ has been observed for other element shapes.

We have observed in the analysis of various reflectarrays that convergence using LegBF is achieved when the largest Floquet harmonic, $k_{\max }$, satisfies the criterium $k_{\max } a=60$, where $a$ is the average spatial support of the basis functions. Consequently the Floquet harmonic summation can be truncated at approximately $N=30 d /(a \pi)$.

The LegBF's ability to model arbitrary-shaped elements together with their good convergence properties make the LegBF well suited for general codes and is an appropriate choice for the analysis of arbitrary reflectarrays.

\section{VALidATION BY MEASUREMENTS}

\section{A. Reference Antenna}

To demonstrate the capabilities of the LegBF, a 900-element reflectarray with a pencil beam toward $\theta=-30^{\circ}$ and $\phi=0^{\circ}$ in the coordinate system shown in Fig. 5 has been designed. It consists of square patches, and its geometrical parameters are summarized in Table I. The feed is a linearly polarized corrugated horn with a taper of $-17.5 \mathrm{~dB}$ at $30^{\circ}$ at $9.6 \mathrm{GHz}$, yielding an illumination along the edges varying from approximately -12 to $-5 \mathrm{~dB}$. The reflectarray and its support structures have been manufactured at the Technical University of Denmark (DTU), Kgs. Lyngby, Denmark, and measured at the DTU-ESA Spherical Near-Field Antenna Test Facility [19]; see Fig. 5. For the peak directivity, the measurements have a $1 \sigma$ uncertainty of $0.07 \mathrm{~dB}$. In addition to the reflectarray measurements, the corrugated horn has also been measured, and the measured data are

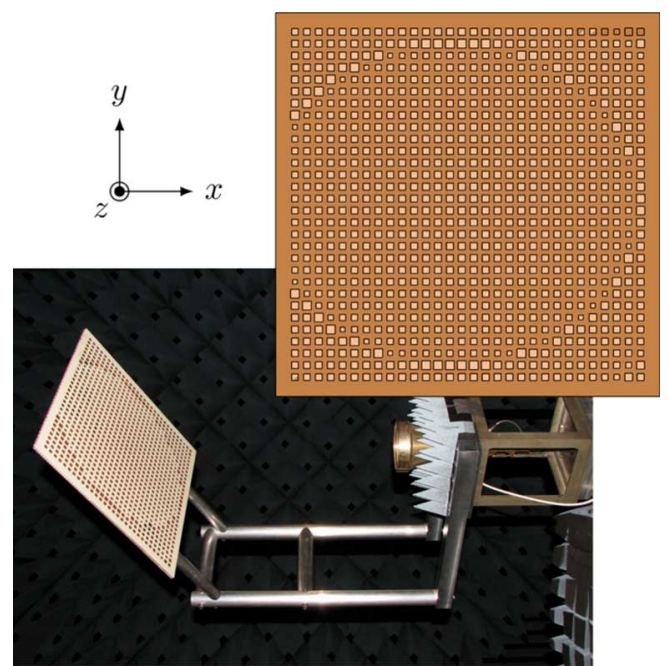

Fig. 5. Reflectarray designed with a pencil beam directed toward $\theta=-30^{\circ}$ and $\phi=0^{\circ}$ in the shown coordinate system and measured at the DTU-ESA Spherical Near-Field Antenna Test Facility.

TABLE I

REFERENCE REFLECTARRAY DATA

\begin{tabular}{l|c}
\hline \hline Frequency & $9.6 \mathrm{GHz}$ \\
\hline Number of elements & $30 \times 30$ \\
\hline Reflectarray dimensions & $435 \mathrm{~mm} \times 435 \mathrm{~mm}$ \\
\hline Substrate thickness & $0.762 \mathrm{~mm}$ \\
\hline Relative permittivity $\epsilon_{\mathrm{r}}$ & 3.66 \\
\hline Loss tangent tan $\delta$ & 0.0037 \\
\hline Feed distance to center of array & $0.6 \mathrm{~m}$ \\
\hline Feed offset angle & $\theta=30^{\circ}, \phi=0^{\circ}$ \\
\hline Main beam direction & $\theta=-30^{\circ}, \phi=0^{\circ}$ \\
\hline \hline
\end{tabular}

used in the SDMoM calculations for accurate representation of the incident field.

\section{B. Simulations Versus Measurements}

For the calculation of the radiation pattern, the continuous spectrum technique from [20, Technique III] is employed. This technique is based on the field equivalence principle and allows the finite extent of the reflectarray to be included. To account for the presence of the support structures, the scattering from the struts is included in the analysis using the MoM add-on in GRASP [21].

The radiation pattern obtained using the E-CheBF and E-LegBF is compared to the measurement results and shown in Fig. 6. The patterns calculated using the two types of basis functions are almost identical. The agreement between the simulated and measured patterns is very good, even for the cross-polar radiation. The discrepancies observed around $\theta=30^{\circ}$ are due to the blockage by the feed and/or the measurement tower.

The analysis of the reference antenna has also been carried out using rooftops, E-sinBF, and S-LegBF, and the performance is summarized in Table II. Due to the thin substrate, and thus highly resonant patches, convergence was not obtained using rooftops, hence the results for the rooftops are omitted in Table II.

This comparison shows that the LegBF is capable of producing results of the same accuracy as those obtained using entire-domain singular basis functions, and for this specific case, 


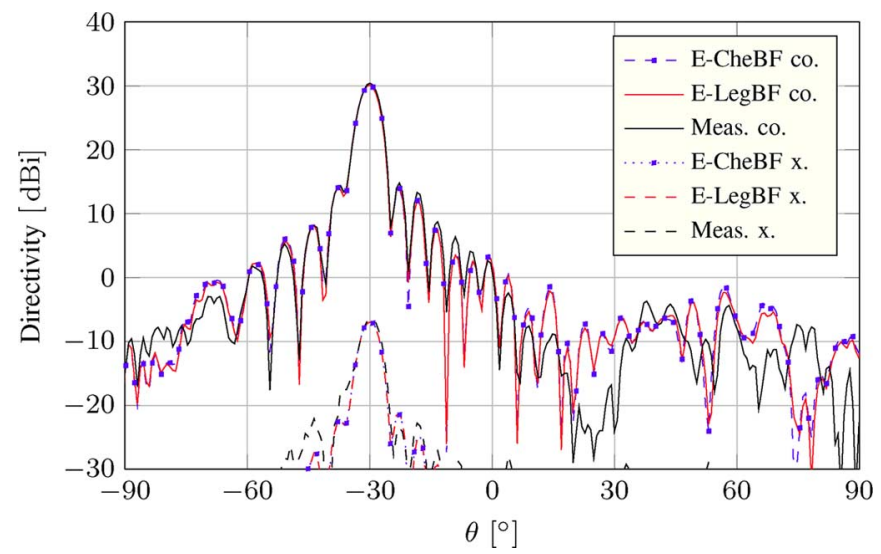

Fig. 6. Simulated and measured co- and cross-polar radiation patterns at $\phi=0^{\circ}$.

TABLE II

Performance of Singular and Higher-Order Hierarchical Legendre BASIS FUNCTIONS

\begin{tabular}{l|c|c|c|c}
\hline \hline & $\begin{array}{c}\text { Peak } \\
\text { Directivity }\end{array}$ & $\begin{array}{c}\text { No. basis } \\
\text { functions }\end{array}$ & $\begin{array}{c}\text { No. Floquet } \\
\text { harmonics }\end{array}$ & $\begin{array}{c}\text { Computation } \\
\text { time }\end{array}$ \\
\hline E-SinBF & $30.1 \mathrm{dBi}$ & 32 & 4141 & $45 \mathrm{~s}$ \\
\hline E-CheBF & $30.1 \mathrm{dBi}$ & 18 & 4141 & $25 \mathrm{~s}$ \\
\hline S-LegBF & $30.1 \mathrm{dBi}$ & 180 & 841 & $1 \mathrm{~min} 35 \mathrm{~s}$ \\
\hline E-LegBF & $30.1 \mathrm{dBi}$ & 84 & 481 & $17 \mathrm{~s}$ \\
\hline Meas. & $30.3 \mathrm{dBi}$ & - & - & - \\
\hline \hline
\end{tabular}

with less computation time, provided no acceleration techniques are used in the SDMoM. For thicker substrates, where a total of only two to eight entire-domain singular basis functions are sufficient for the accurate characterization of the array elements, approximately 24-40 E-LegBF are required, and the computation times for the two cases are practically identical.

\section{CONCLUSION}

The use of higher-order hierarchical Legendre basis functions for the analysis of printed reflectarrays has been presented. The higher-order hierarchical Legendre basis functions can be applied to arbitrarily shaped array elements and have better convergence rate compared to first-order basis functions, e.g., rooftop basis functions. For elements that can be described using a single mesh element, the higher-order hierarchical Legendre basis functions can be defined on the entire patch, thus becoming entire-domain basis functions. For these cases, where singular basis functions are known for accurately accounting for the singularities of the electric current on the patch edges, it is demonstrated that the higher-order hierarchical Legendre basis functions are capable of producing results of the same accuracy as those obtained using singular basis functions. In addition, higher-order hierarchical Legendre basis functions require less Floquet harmonics than singular basis functions, thus a good compromise between the number of Floquet harmonics and unknowns can be obtained using higher-order hierarchical Legendre basis functions.

An offset reflectarray has been designed, manufactured, and measured to serve as a reference. The agreement with measurements is extremely good, thus demonstrating the capabilities of the higher-order hierarchical Legendre basis functions.

\section{ACKNOWLEDGMENT}

Dr. S. Pivnenko, Technical University of Denmark, is acknowledged for the measurements of the reflectarray sample.

\section{REFERENCES}

[1] J. Huang and J. A. Encinar, Reflectarray Antennas. Piscataway, NJ: IEEE Press, 2008.

[2] D. M. Pozar, S. D. Targonski, and H. D. Syrigos, "Design of millimeter wave microstrip reflectarrays," IEEE Trans. Antennas Propag., vol. 45, no. 2, pp. 287-296, Feb. 1997.

[3] R. Mittra, C. H. Chan, and T. Cwik, "Techniques for analyzing frequency selective surfaces-A review," Proc. IEEE, vol. 76, no. 12, pp. 1593-1615, Dec. 1988.

[4] W. C. Chew and Q. Liu, "Resonance frequency of a rectangular microstrip patch," IEEE Trans. Antennas Propag., vol. 36, no. 8, pp. 1045-1056, Aug. 1988.

[5] A. M. Lerer and A. G. Schuchinsky, "Full-wave analysis of three-dimensional planar structures," IEEE Trans. Microw. Theory Tech., vol. 41, no. 11, pp. 2002-2015, Nov. 1993.

[6] S. M. Rao, D. R. Wilton, and A. W. Glisson, "Electromagnetic scattering by surfaces of arbitrary shape," IEEE Trans. Antennas Propag., vol. AP-30, no. 3, pp. 409-418, May 1982.

[7] A. W. Glisson and D. R. Wilton, "Simple and efficient numerical methods for problems of electromagnetic radiation and scattering from surfaces," IEEE Trans. Antennas Propag., vol. AP-28, no. 5, pp. 593-603, Sep. 1980.

[8] E. Jørgensen, J. Volakis, P. Meincke, and O. Breinbjerg, "Higher order hierarchical Legendre basis functions for electromagnetic modeling," IEEE Trans. Antennas Propag., vol. 52, no. 11, pp. 2985-2995, Nov. 2004.

[9] S. R. Rengarajan, "Choice of basis functions for accurate characterization of infinite array of microstrip reflectarray elements," IEEE Antennas Wireless Propag. Lett., vol. 4, pp. 47-50, 2005.

[10] R. R. Boix, M. J. Freire, and F. Medina, "New method for the efficient summation of double infinite series arising from the spectral domain analysis of frequency selective surfaces," IEEE Trans. Antennas Propag., vol. 52, no. 4, pp. 1080-1094, Apr. 2004.

[11] G. Cano, F. Medina, and M. Horno, "On the efficient implementation of SDA for boxed strip-like and slot-like structures," IEEE Trans. Microw. Theory Tech., vol. 46, no. 11, pp. 1801-1806, Nov. 1998.

[12] S.-O. Park, C. A. Balanis, and C. R. Birtcher, "Analytical evaluation of the asymptotic impedance matrix of a grounded dielectric slab with roof-top functions," IEEE Trans. Antennas Propag., vol. 46, no. 2, pp. 251-259, Feb. 1998

[13] M. R. Chaharmir, J. Shaker, N. Gagnon, and D. Lee, "Design of broadband, single layer dual-band large reflectarray using multi open loop elements," IEEE Trans. Antennas Propag., vol. 58, no. 9, pp. 2875-2883, Sep. 2010.

[14] M. E. Bialkowski and K. H. Sayidmarie, "Investigations into phase characteristics of a single-layer reflectarray employing patch or ring elements of variable size," IEEE Trans. Antennas Propag., vol. 56, no. 11, pp. 3366-3372, Nov. 2008.

[15] C. Han, C. Rodenbeck, J. Huang, and K. Chang, "A C/Ka dual frequency dual layer circularly polarized reflectarray antenna with microstrip ring elements," IEEE Trans. Antennas Propag., vol. 52, no. 11, pp. 2871-2876, Nov. 2004.

[16] D. Cadoret, A. Laisne, R. Gillard, and H. Legay, "A new reflectarray cell using microstrip patches loaded with slots," Microw. Opt. Technol. Lett., vol. 44, no. 3, pp. 270-272, 2005.

[17] P. De Vita, A. Freni, G. L. Dassano, P. Pirinoli, and R. E. Zich, "Broadband element for high-gain single-layer printed reflectarray antenna," Electron. Lett., vol. 43, no. 23, pp. 1247-1249, 2007.

[18] L. Moustafa, R. Gillard, F. Peris, R. Loison, H. Legay, and E. Girard, "The phoenix cell: A new reflectarray cell with large bandwidth and rebirth capabilities," IEEE Antennas Wireless Propag. Lett., vol. 10, pp. 71-74, 2011

[19] Technical University of Denmark, Kgs. Lyngby, Denmark, "DTU-ESA spherical near-field antenna test facility," 2011 [Online]. Available: http://www.dtu.dk/centre/ems/English/research/facilities.aspx

[20] M. Zhou, S. B. Sørensen, E. Jørgensen, P. Meincke, O. S. Kim, and O. Breinbjerg, "An accurate technique for calculation of radiation from printed reflectarrays," IEEE Antennas Wireless Propag. Lett., vol. 10, pp. 1081-1084, 2011

[21] K. Pontoppidan, Ed., "GRASP, Technical description,” TICRA Engineering Consultants, Copenhagen, Denmark, 2008. 